\title{
New Structure of Fuzzy Fully Invariant Bipolar Q-Fuzzy Regular Lattices
}

\author{
A.Solairaju and A.Prasanna \\ Department of Mathematics \\ Jamal Mohamed College \\ Tiruchirappalli -620020 \\ Tamilnadu, India
}

\begin{abstract}
In this paper we introduced the notion of interval-valued bipolar Q-fuzzy lattices, briefly IVBQFL, in terms of fuzzy dideals based on bipolar valued fuzzy set and several related properties are also established. Relations between a intervalvalued bipolar Q-fuzzy lattices and bipolar fuzzy d-ideals are also discussed. The concept of Cartesian product fuzzy fully invariant and characteristic of interval-valued bipolar Q-fuzzy lattices are investigated. The homomorphic image of bipolar Q-fuzzy lattice is also given. Furthermore, we state family of interval-valued bipolar Q-fuzzy lattices.
\end{abstract}

AMS Mathematics subject classification (2000):06F35, 03G25, 08A72, 03E72

Key Words: Bipolar Q-fuzzy lattice, Interval-valued bipolar Q-fuzzy lattice, Cartesian product, Fuzzy Fully Invariant, Characteristic

\section{INTRODUCTION}

In the traditional fuzzy sets, the membership degrees of elements range over the interval $[0,1]$. Bipolar valued fuzzy sets and intuitionistic fuzzy sets where introduced in 1986 only with the membership degrees ranged on the interval $[0$, 1]. It is difficult to express the difference of the irrelevant elements from the contrary elements in fuzzy set. If a set representation could express this kind of difference, it would be more informative than the traditional fuzzy set representation. Based on these observations, K.M. Lee [7], introduced an extention of fuzzy sets named bipolar-valued fuzzy sets. He give two kinds of representations of the notion of bipolar-valued fuzzy sets in. Jun and Song [5] applied the notion of bipolar-valued fuzzy sets to $\mathrm{BCH}$-Algebras. They introduced the concept of bipolar fuzzy sub algebras / ideals of a $\mathrm{BCH}$ algebras and investigated several properties. Interval-valued fuzzy sets were first introduced by the Zadeh [11] as a generalization of fuzzy sets. This idea gives the simplest method to capture the imprecision of the membership grades for a fuzzy sets. Thus interval-valued fuzzy set provide a more adequate description of uncertainity then the traditional fuzzy sets. It is therefore important to use intervalvalued fuzzy sets in applications. One of the main application is in fuzzy control and the most computationally intensive part of fuzzy control is defuzzification. With the above background Atanassov and Gargov [1] introduce the notion of interval-valued intuitionistic fuzzy sets, which is a common generalization of intuitionstic fuzzy sets and interval-valued fuzzy sets. Therefore fuzzy sets are a kind of useful Mathematical structure to represent a collection of objects whose boundary is vague. There are several kinds of fuzzy set extensions in the fuzzy set theory, for example, intuitionistic fuzzy sets, interval-valued fuzzy sets, vague set etc. Bipolar valued fuzzy sets are an extension of fuzzy sets whose membership degree range is enlarged from the interval $[0,1]$ to $[-1,1]$. Bipolar valued fuzzy set have membership degrees that represent the degree of satisfaction to the property corresponding to a fuzzy set and its counter property. In a bipolar-valued fuzzy set, the membership degree 0 means that elements are irrelevant to the corresponding property, the membership degrees on $[0,1]$ indicate that elements somewhat satisfy the property, and the membership degrees on $[-1,0]$ indicate that elements somewhat satisfy the implicit counter-property (see [8]).

Figure 1 shows a bipolar-valued fuzzy set redefined for the fuzzy set "young" .. The negative membership degrees indicate the satisfaction extent of elements to an implicit counter-property. (e.g. old against the property young). This kind of bipolar-valued fuzzy set representation enables the elements with membership degree 0 in traditional fuzzy sets, to be expressed into the elements with membership degree 0 (irrelevant elements). The age elements 50 and 95, with membership degree 0 in the fuzzy sets of figure 1 , have 0 and a negative membership degree in the bipolar-valued fuzzy set of figure 1, respectively. Now it is manifested that 50 is an irrelevant age to the property young and 95 is more apart from the property young than 50 , i.e. 95 is a contrary age to the property young see[8]

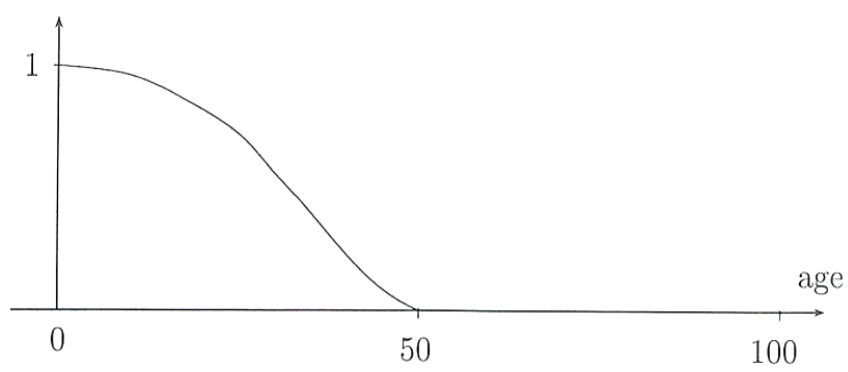

Figure 1. A bipolar fuzzy set "young"

In this paper, we introduce the notion of interval-valued bipolar fuzzy lattice, Cartesian product, fuzzy fully invariant lattice, characteristic and a homomorphic image of bipolar fuzzy lattice and then we investigate several properties. We give a characterization of bipolar fuzzy d-ideals. 


\section{PRELIMINARIES}

Definition 2.1 :Let X be a non-empty set. A Q-fuzzy set in X is function $\mu: \mathrm{X} \times \mathrm{Q} \rightarrow[0,1]$

Definition 2.2 : Let $G$ be a any non-empty set. A bipolar valued Q-fuzzy set $A$ in $G$ is an object having the form $\mathrm{A}=\left\{\left(\mathrm{x}, \mu_{\mathrm{A}}^{+}(\mathrm{x}, \mathrm{q}), \mu_{\mathrm{A}}^{-}(\mathrm{x}, \mathrm{q})\right) / \mathrm{x} \in \mathrm{G}\right\}$ Where $\mu_{\mathrm{A}}^{+}: \mathrm{G} \times \mathrm{Q} \rightarrow[0$, 1] and $\mu_{\mathrm{A}}^{-}: \mathrm{G} \times \mathrm{Q} \rightarrow[-1,0]$. are mapping. The positive membership degree $\mu_{\mathrm{A}}^{-}(\mathrm{x}, \mathrm{q})$ denotes the satisfaction degree of an element $\mathrm{x}$ to the property corresponding to " $\mathrm{A}$ " and the negative membership degree $\mu_{\mathrm{A}}^{-}(\mathrm{x}, \mathrm{q})$ denotes the satisfaction degree of $\mathrm{x}$ to some implicit counter property of A.

Definition 2.3 :A Bipolar Lattice fuzzy set $A$ in $G$ is called Bipolar Q-fuzzy Lattice of $\mathrm{G}$ if it satisfies

(BFQL1)

$\mu^{+}(x+y, q) \geq \min \left\{\mu^{+}(x, q), \mu^{+}(y, q)\right.$

(BFQL2)

$\mu^{-}(\mathrm{x}+\mathrm{y}, \mathrm{q}) \leq \max \left\{\mu^{-}(\mathrm{x}, \mathrm{q}), \mu^{-}(\mathrm{y}, \mathrm{q})\right\}$

$(\operatorname{BFQL3}) \mu^{+}(-\mathrm{x}, \mathrm{q}) \geq \mu^{-}(\mathrm{x}, \mathrm{q})$ and $\quad \mu^{-}(\mathrm{x}, \mathrm{q}) \leq \mu^{-}(\mathrm{x}, \mathrm{q})$

(BFQL4)

$\mu^{+}(\mathrm{x} \mathrm{y}, \mathrm{q}) \geq \min \left\{\mu^{+}(\mathrm{x}, \mathrm{q}), \mu^{+}(\mathrm{y}, \mathrm{q})\right\}$

\section{(BFQL5)}

$\mu^{-}(x y, q) \leq \max \left\{\mu^{-}(x, q), \mu^{-}(y, q)\right\}$, for all $x, y \in G$.

Definition 2.4: A Lattice ordered Group (LG) is a system $\mathrm{G}=$ $(\mathrm{G},+, \leq)$

(i) $(\mathrm{G},+)$ is a group $(\mathrm{ii})(\mathrm{G}, \cdot)$ is a lattice.

Definition 2.5: For a Bipolar Q-fuzzy set $A$ in and $G(\beta, \alpha) \in$ $[-1,0] \times[0,1]$.

We define $\mathrm{A}_{\mathrm{t}}^{+}=\left\{\mathrm{x} \in \mathrm{X} / \mu_{\mathrm{A}}^{+}(\mathrm{x}, \mathrm{q}) \leq \alpha\right\}, \mathrm{A}_{\mathrm{S}}^{-}=\left\{\mathrm{x} \in \mathrm{X} / \mu_{\mathrm{A}}\right.$ $(\mathrm{x}, \mathrm{q}) \geq \alpha\}$ Which are called positive t-cut and Negative s-cut of A respectively.

Definition 2.6 :Let A be a Bipolar Lattice over a group G with $[\mathrm{x}, \mathrm{y}] \in \mathrm{A}$. Then $\mathrm{A}$ is called interval-valued Bipolar Qfuzzy lattice over $\mathrm{G}$ if

$(\operatorname{IVBFQL1}) \mu^{+}(\mathrm{x}+\mathrm{y}, \mathrm{q}) \geq \min \left\{\mu^{+}(\mathrm{x}, \mathrm{q}), \mu^{+}(\mathrm{y}, \mathrm{q})\right\}$

$(\operatorname{IVBFQL2}) \mu^{-}(\mathrm{x}+\mathrm{y}, \mathrm{q}) \leq \max \left\{\mu^{-}(\mathrm{x}, \mathrm{q}), \mu^{-}(\mathrm{y}, \mathrm{q})\right\}$

$(\operatorname{IVBFQL3}) \mu^{+}(-\mathrm{x}, \mathrm{q}) \geq \mu^{-}(\mathrm{x}, \mathrm{q})$ and $\mu^{-}(\mathrm{x}, \mathrm{q}) \leq \mu^{-}(\mathrm{x}, \mathrm{q})$

$(\operatorname{IVBFQL} 4) \mu^{+}[\mathrm{x}, \mathrm{y}]_{\mathrm{q}} \geq \min \left\{\mu^{+}(\mathrm{x}, \mathrm{q}), \mu^{+}(\mathrm{y}, \mathrm{q})\right\}$

$\left(\right.$ IVBFQL5) $\mu^{-}[\mathrm{x}, \mathrm{y}]_{\mathrm{q}} \leq \max \left\{\mu^{-}(\mathrm{x}, \mathrm{q}), \mu^{-}(\mathrm{y}, \mathrm{q})\right\}$, for all $\mathrm{x}, \mathrm{y} \in$ $\mathrm{G}$ and $\mathrm{q}$ in $\mathrm{Q}$.

Definition 2.7: A Bipolar Q- fuzzy set (BQFS) A in G is called Bipolar Q-fuzzy d-ideal of $\mathrm{G}$ if it satisfies

$$
\begin{array}{ll}
(B F Q D 1) \mu^{+}(\mathrm{x}, \mathrm{q}) \geq \min \left\{\mu^{+}(\mathrm{xy}, \mathrm{q}),\right. & \left.\mu^{+}(\mathrm{y}, \mathrm{q})\right\} \\
(\mathrm{BFQD} 2) \mu^{-}(\mathrm{x}, \mathrm{q}) \leq \max \left\{\mu^{-}(\mathrm{xy}, \mathrm{q}),\right. & \left.\mu^{-}(\mathrm{y}, \mathrm{q})\right\} \\
(\mathrm{BFQD} 3) \mu^{+}[\mathrm{x}, \mathrm{y}]_{\mathrm{q}} \leq \min \left\{\mu^{+}(\mathrm{x}, \mathrm{q}),\right. & \left.\mu^{+}(\mathrm{y}, \mathrm{q})\right\} \\
(\mathrm{BFQD} 4) \mu^{-}[\mathrm{x}, \mathrm{y}]_{\mathrm{q}} \geq \max \left\{\mu^{-}(\mathrm{x}, \mathrm{q}),\right. & \left.\mu^{-}(\mathrm{y}, \mathrm{q})\right\}, \text { for all } \mathrm{x},
\end{array}
$$
$\mathrm{y} \in \mathrm{G}$ and $\mathrm{q}$ in $\mathrm{Q}$.

Definition 2.8: Let $\lambda$ and $\mu$ be two fuzzy subset in $X$. The Cartesian product

$\lambda^{+} \times \mu^{+}: \mathrm{X} \times \mathrm{X} \rightarrow[0,1]$ is defined by $\lambda^{+} \times \mu^{+}(\mathrm{x}, \mathrm{y})=\mathrm{T}\left\{\lambda^{+}\right.$ $\left.(\mathrm{x}), \mu^{+}(\mathrm{y})\right\} \quad$ and $\lambda^{-} \times \mu^{-}(\mathrm{x}, \mathrm{y})=\mathrm{S}\left\{\lambda^{-}(\mathrm{x}), \mu^{-}(\mathrm{y})\right\} \quad$ for all $\mathrm{x}, \mathrm{y} \in \mathrm{G}$.

Definition 2.9: An Interval-valued Bipolar Q-fuzzy d-ideal A of $L$ is Fuzzy fully invariant if $\mu_{1}^{+f}(x, q)=\mu^{+f}(x, q)$ and $\mu_{2}^{-f}$ $(\mathrm{x}, \mathrm{q})=\mu^{-\mathrm{f}}(\mathrm{x}, \mathrm{q})$

Definition 2.10: Let A be a Bipolar Q-Fuzzy set in X. The strongest Bipolar Q-fuzzy relation on $\mathrm{X}$ that is fuzzy relation on $\mathrm{A}$ is $\mu_{\mathrm{A}}$ given by $\mu_{\mathrm{A}}^{+}(\mathrm{x}, \mathrm{y})_{\mathrm{q}}=\mathrm{T}\left\{\mathrm{A}^{+}(\mathrm{x}, \mathrm{q}), \mathrm{A}^{+}(\mathrm{y}, \mathrm{q})\right\}$.

$\mu_{\mathrm{A}}^{-}(\mathrm{x}, \mathrm{y})_{\mathrm{q}}=\mathrm{S}\left\{\mathrm{A}^{-}(\mathrm{x}, \mathrm{q}), \mathrm{A}^{-}(\mathrm{y}, \mathrm{q})\right\}$ for all $\mathrm{x}, \mathrm{y} \in \mathrm{G}$. For a sake of simplicity we shall use the symbol. $A=\left\{\mu^{+}, \mu^{-}\right\}$for the bipolar valued Q-fuzzy set $A=\left\{\left(\left(x, \mu^{+}(x, q), \mu^{-}(x, q)\right) / x \in\right.\right.$ $\mathrm{G}$ ) and for bipolar fuzzy regular lattice (BFRL) of G.

\section{PROPERTIES OF BIPOLAR Q-}

\section{FUZZY LATTICES}

Proposition 3.1 : If all non-empty level subsets $U(A ; \alpha)$ of an interval-valued bipolar Q-fuzzy set are bipolar-Q- fuzzy dideals then A is an interval valued Bipolar Q-fuzzy Lattice.

Proof : Let $\alpha \in[0,1]$. Suppose that $\mathrm{U}(\mathrm{A} ; \alpha) \neq \phi$ are fuzzy ideals in A. We must show that A satisfies the conditions of definition 2.6 of IVBQFL1 - IVBQFL5. If the condition IVBQFL1 is false the there exist $\mathrm{x}, \mathrm{y} \in \mathrm{A}$, such that $\mu^{+}(\mathrm{x}, \mathrm{y})_{\mathrm{q}} \geq \min \left\{\mu^{+}(\mathrm{x}, \mathrm{q}), \mu^{+}(\mathrm{y}, \mathrm{q})\right\}$ Taking

$\mu^{+}(x, y)_{q}+\min \left\{\mu^{+}(x, q), \mu^{+}(y, q)\right.$ $\mathrm{t}_{0}=$

We have, $\mu^{+}(\mathrm{x}+\mathrm{y}, \mathrm{q}) \leq 2 \mathrm{t}_{0} \leq \min \left\{\mu^{+}(\mathrm{x}, \mathrm{q}), \mu^{+}(\mathrm{y}, \mathrm{q})\right\}$.It follows that $\mathrm{x}+\mathrm{y} \notin \mathrm{U}(\mathrm{A}, \alpha)$ and $\mathrm{x}, \mathrm{y} \in \mathrm{U}(\mathrm{A}, \alpha)$ Which is a contradiction. Hence the conditions of definition 2.6 is true.

Similarly, we can show IVBQFL 2

- IVBQFL 5.Hence A is interval valued Bipolar Q- fuzzy lattice over G.

Proposition 3.2:Let $\left\{\mu_{\mathrm{i}}: \mathrm{i} \in \Lambda\right\}$ be a family of interval valued bipolar Q-fuzzy lattice $\mathrm{L}$ over groups $\mathrm{G}$ such that $\mu_{\mathrm{i}}^{+}$ 
$\subseteq \mu_{\mathrm{j}}^{+}$or $\quad \mu_{\mathrm{i}}^{-} \subseteq \mu_{\mathrm{j}}^{-} \quad$ for all $\mathrm{i}, \mathrm{j} \in \mathrm{A}$. Then $\gamma=\bigcup_{\mathrm{i} \in \Lambda} \gamma_{\mathrm{i}}$ is an interval Valued bipolar Q- fuzzy lattice over a group G.

Proof :Assume $\left\{\mu_{\mathrm{i}}: \mathrm{i} \in \Lambda\right\}$ be a family of bipolar Q-fuzzy lattice over G. We have (IVBQFL1) $\mu^{+}(\mathrm{x}+\mathrm{y}, \mathrm{q}) \quad \geq \min$ $\left\{\mu^{+}(\mathrm{x}, \mathrm{q}), \mu^{+}(\mathrm{y}, \mathrm{q})\right\}$

And hence

$\mu^{+}(x+y, q) \quad=\inf \left\{\mu_{i}^{+}(x+y, q)\right\}$

is $\Lambda$

$\geq \inf \left\{\min \left\{\mu_{\mathrm{i}}^{+}(\mathrm{x}, \mathrm{q}), \mu_{\mathrm{i}}^{+}(\mathrm{y}, \mathrm{q})\right\}\right.$

i $\varepsilon \Lambda$

$=\min \left\{\inf \mu_{\mathrm{i}}^{+}(\mathrm{x}, \mathrm{q}), \quad \inf \mu_{\mathrm{i}}^{+}(\mathrm{y}, \mathrm{q})\right\}$

$=\min \left\{\bigcup_{\mathrm{i} \in \Lambda} \mu_{\mathrm{i}}^{+}(\mathrm{x}, \mathrm{q}), \bigcup_{\mathrm{i} \in \Lambda} \mu_{\mathrm{i}}^{+}(\mathrm{y}, \mathrm{q}),\right\}$

$=\min \left\{\mu^{+}(\mathrm{x}, \mathrm{q}), \mu^{+}(\mathrm{y}, \mathrm{q})\right\}$

It is easy to see that

$\inf \left[\min \left\{\mu_{\mathrm{i}}{ }^{+}(\mathrm{x}, \mathrm{q}), \mu_{\mathrm{i}}{ }^{+}(\mathrm{y}, \mathrm{q})\right\}\right] \leq \bigcup_{\mathrm{i} \in \Lambda}\left[\min \left\{\mu_{\mathrm{i}}^{+}(\mathrm{x}, \mathrm{q}), \mu_{\mathrm{i}}^{+}\right.\right.$ $(\mathrm{y}, \mathrm{q})\}]$

Suppose that

$\inf \left[\min \left\{\mu_{\mathrm{i}}{ }^{+}(\mathrm{x}, \mathrm{q}), \mu_{\mathrm{i}}{ }^{+}(\mathrm{y}, \mathrm{q})\right\}\right] \neq \bigcup_{\mathrm{i} \in \Lambda}\left[\min \left\{\mu_{\mathrm{i}}^{+}(\mathrm{x}, \mathrm{q}), \mu_{\mathrm{i}}^{+}\right.\right.$ $(y, q)\}]$

Since

$$
\mu_{\mathrm{i}}^{+} \subseteq \mu_{\mathrm{j}}^{+} \text {or } \quad \mu_{\mathrm{i}}^{-} \subseteq \mu_{\mathrm{j}}^{-} \quad \text { for all } \mathrm{i}, \mathrm{j} \in \Lambda
$$

There exist $\mathrm{C} \in \Lambda$, such that $\mathrm{t}_{0} \leq \min \left\{\mu_{\mathrm{c}}^{+}(\mathrm{x}, \mathrm{q}), \mu_{\mathrm{c}}^{+}(\mathrm{y}, \mathrm{q})\right\}$

On the other hand,

$\min \left\{\mu_{\mathrm{i}}{ }^{+}(\mathrm{x}, \mathrm{q}), \mu_{\mathrm{i}}{ }^{+}(\mathrm{y}, \mathrm{q})\right\} \geq \mathrm{t}_{0}$, for $\mathrm{i}, \mathrm{j} \in \Lambda$. Which is a contradiction. Therefore,

$\inf \left[\min \left\{\mu_{\mathrm{i}}^{+}(\mathrm{x}, \mathrm{q}), \mu_{\mathrm{i}}^{+}(\mathrm{y}, \mathrm{q})\right\}\right] \geq \min \left\{\mu^{+}(\mathrm{x}, \mathrm{q}), \mu^{+}\right.$

$(\mathrm{y}, \mathrm{q})\}$

i $\varepsilon \Lambda$

(IVBQFL2) $\mu^{-}(x+y, q) \leq \max \left\{\mu^{-}(x, q), \mu^{-}(y, q)\right\}$

Now

$\mu^{-}(\mathrm{x}+\mathrm{y}, \mathrm{q})=\sup \left\{\mu^{-}(\mathrm{x}+\mathrm{y}, \mathrm{q})\right\}$

i $\varsigma \Lambda$

$\leq \sup \left\{\max \left\{\mu_{\mathrm{i}}^{-}(\mathrm{x}, \mathrm{q}), \mu_{\mathrm{i}}^{-}(\mathrm{y}, \mathrm{q})\right\}\right\}$

i $\varepsilon \Lambda$

$=\max \left\{\sup \mu_{\mathrm{i}}^{-}(\mathrm{x}, \mathrm{q}), \sup \mu_{\mathrm{i}}^{-}(\mathrm{y}, \mathrm{q})\right\}$

i $\varepsilon \Lambda \quad$ i $\Lambda \Lambda$ $=\max \left\{\bigcap_{\mathrm{i} \in \Lambda} \mu_{\mathrm{i}}^{-}(\mathrm{x}, \mathrm{q}), \bigcap_{\mathrm{i} \in \Lambda} \mu_{\mathrm{i}}^{-}(\mathrm{y}, \mathrm{q})\right\}$

$=\max \left\{\mu^{-}(\mathrm{x}), \mu^{-}(\mathrm{y})\right\}$

It is easy to see that

$\sup \left[\max \left\{\left\{\mu_{\mathrm{i}}^{-}(\mathrm{x}, \mathrm{q}), \mu_{\mathrm{i}}^{-}(\mathrm{y}, \mathrm{q})\right\}\right] \geq \bigcap_{\mathrm{i} \in \Lambda}\left[\max \left\{\mu_{\mathrm{i}}^{-}(\mathrm{x}, \mathrm{q})\right.\right.\right.$,

$\left.\left.\mu_{\mathrm{i}}^{-}(\mathrm{y}, \mathrm{q})\right\}\right]$

Suppose that

$\sup \left[\max \left\{\left\{\mu_{\mathrm{i}}^{-}(\mathrm{x}, \mathrm{q}), \mu_{\mathrm{i}}^{-}(\mathrm{y}, \mathrm{q})\right\}\right] \neq \bigcap_{\mathrm{i} \in \Lambda}\left[\max \left\{\mu_{\mathrm{i}}^{-}(\mathrm{x}, \mathrm{q}), \mu\right.\right.\right.$

$\left.\left.\mathrm{i}^{-}(\mathrm{y}, \mathrm{q})\right\}\right]$

then there exist a $t_{0}$ such that $\sup \left[\max \left\{\mu_{\mathrm{i}}^{-}(\mathrm{x}, \mathrm{q}), \mu_{\mathrm{i}}^{-}(\mathrm{y}, \mathrm{q})\right\}\right] \leq \mathrm{t}_{0} \leq \bigcap_{\mathrm{i} \in \Lambda}[\max$

$\left.\left\{\mu_{\mathrm{i}}^{-}(\mathrm{x}, \mathrm{q}), \mu_{\mathrm{i}}^{-}(\mathrm{y}, \mathrm{q})\right\}\right]$

is $\Lambda$

Since $\quad \mu_{\mathrm{i}}^{-} \subseteq \mu_{\mathrm{j}}^{-}$or $\mu_{\mathrm{i}}^{+} \subseteq \mu_{\mathrm{j}}^{+} \quad$ for all $\mathrm{i}, \mathrm{j} \in \Lambda$

Therefore, there exist $\mathrm{C} \in \Lambda$ such that $\mathrm{t}_{\mathrm{o}} \geq \max \left\{\mu_{\mathrm{c}}^{-}(\mathrm{x}, \mathrm{q})\right.$, $\left.\left.\mu_{\mathrm{c}}^{-}(\mathrm{y}, \mathrm{q})\right\}\right]$

on the other hand, $\max \left\{\mu_{\mathrm{c}}^{-}(\mathrm{x}, \mathrm{q}), \quad \mu_{\mathrm{c}}^{-}(\mathrm{y}, \mathrm{q})\right\} \leq \mathrm{t}_{\mathrm{o}}$, for $\mathrm{i} \in \Lambda$.Which is a contradiction. Therefore.

$\sup \left[\max \left\{\mu_{\mathrm{i}}^{-}(\mathrm{x}, \mathrm{q}), \mu_{\mathrm{i}}^{-}(\mathrm{y}, \mathrm{q})\right\}\right] \leq \max \left\{\mu_{\mathrm{i}}^{-}(\mathrm{x}, \mathrm{q}), \mu_{\mathrm{i}}{ }^{-}\right.$ $(\mathrm{y}, \mathrm{q})\}]$

(IVBQFL3), $\mu^{+}(-\mathrm{x}, \mathrm{q})$

$=\inf \left\{\mu_{\mathrm{i}}^{+}(-\mathrm{x}, \mathrm{q})\right\} \geq \inf \left\{\mu_{\mathrm{i}}^{+}(\mathrm{x}, \mathrm{q})\right\}$

is $\Lambda$

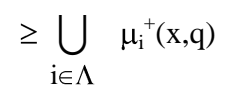

$\geq \mu^{+}(\mathrm{x}, \mathrm{q})$ and

$\mu^{-}(-\mathrm{x}) \quad=\sup \left\{\mu_{\mathrm{i}}^{-}(-\mathrm{x}, \mathrm{q})\right\}$

ic $\Lambda$

$\geq \sup \left\{\mu_{\mathrm{i}}^{-}(\mathrm{x}, \mathrm{q})\right\}$

i $\Lambda$

$$
\begin{aligned}
& \geq \bigcap_{i \in \Lambda} \mu_{\mathrm{i}}^{-}(\mathrm{x}, \mathrm{q}) \\
& \geq \mu^{-}(\mathrm{x}, \mathrm{q})
\end{aligned}
$$

(IVBQFL4), We have

$\mu^{+}[x, y] q=\min \left\{\mu^{+}(x, q), \mu^{+}(y, q)\right\}$ and hence

$$
\mu^{+}[x, y] q=\inf \mu_{\mathrm{i}}^{+}[x, y] q
$$

i $\varepsilon \Lambda$

$\geq \inf \min \left\{\mu_{\mathrm{i}}^{+}(\mathrm{x}, \mathrm{q}), \mu_{\mathrm{i}}^{+}(\mathrm{y}, \mathrm{q})\right\}$

is $\Lambda$

$\left.\geq \min \left[\inf \mu_{\mathrm{i}}^{+}(\mathrm{x}, \mathrm{q}), \inf \mu_{\mathrm{i}}^{+}(\mathrm{y}, \mathrm{q})\right\}\right]$ 


$$
\begin{aligned}
& \quad i \varepsilon \Lambda \quad \operatorname{iic\Lambda } \\
& \left.\geq \min \left\{\bigcup_{\mathrm{i} \in \Lambda} \mu_{\mathrm{i}}^{+}(\mathrm{x}, \mathrm{q}), \bigcup_{\mathrm{i} \in \Lambda} \mu_{\mathrm{i}}^{+}(\mathrm{y}, \mathrm{q})\right\}\right\} \\
& \geq \min \left\{\mu^{+}(\mathrm{x}, \mathrm{q}), \mu^{+}(\mathrm{y}, \mathrm{q})\right\} \\
& (\operatorname{IVBQFL} 5) \mu^{-}[\mathrm{x}, \mathrm{y}]_{\mathrm{q}}=\sup \mu_{\mathrm{i}}^{-}([\mathrm{x}, \mathrm{y}] \mathrm{q}) \\
& \leq \sup \left[\max \left\{\mu_{\mathrm{i}}^{-}(\mathrm{x}, \mathrm{q}), \mu_{\mathrm{i}}^{-}(\mathrm{y}, \mathrm{q})\right\}\right] \\
& \left.\quad \operatorname{i\varepsilon \Lambda } \max \left[\sup \mu_{\mathrm{i}}^{-}(\mathrm{x}, \mathrm{q}), \sup \mu_{\mathrm{i}}^{-}(\mathrm{y}, \mathrm{q})\right\}\right] \\
& \left.\quad \operatorname{i\varepsilon \Lambda } \quad \max \left\{\bigcap_{\mathrm{i} \in \Lambda} \mu_{\mathrm{i}}^{-}(\mathrm{x}, \mathrm{q}), \bigcap_{\mathrm{i} \in \Lambda} \mu_{\mathrm{i}}^{-}(\mathrm{y}, \mathrm{q})\right\}\right\} \\
& \leq \max \left\{\mu^{-}(\mathrm{x}, \mathrm{q}), \mu^{-}(\mathrm{y}, \mathrm{q})\right\}
\end{aligned}
$$

Therefore $\mathrm{A}$ is an interval valued bipolar Q-fuzzy lattice over G.

Proposition 3.3 : Let $\mathrm{A}=\left(\mathrm{X}, \mu_{\mathrm{A}}^{+}, \mu_{\overline{\mathrm{A}}}\right)$ be a interval valued bipolar Q-fuzzy d-ideal of X. If the inequality $\mathrm{x} \times \mathrm{y} \leq \mathrm{z}$ holds in $\mathrm{X}$ then.

$$
\begin{aligned}
& \text { (i) } \mu_{\mathrm{A}}^{+} \quad(\mathrm{x}, \mathrm{q}) \geq \min \quad\left\{\mu_{\mathrm{A}}^{+}(\mathrm{y}, \mathrm{q}), \mu_{\mathrm{A}}^{+}(\mathrm{z}, \mathrm{q})\right\}(\text { ii) }) \mu_{\mathrm{A}} \quad(\mathrm{x}, \mathrm{q}) \\
& \leq \max \left\{\mu_{\mathrm{A}}(\mathrm{y}, \mathrm{q}), \mu_{\mathrm{A}}(\mathrm{z}, \mathrm{q})\right\}
\end{aligned}
$$

Proof :Let $x, y, z \in X$ be such that $x y \leq z$ then $(x y) z=0$ and so

$$
\begin{aligned}
& \text { (i) } \mu_{\mathrm{A}}^{+}(\mathrm{x}, \mathrm{q}) \quad \geq \min \left\{\mu_{\mathrm{A}}^{+}(\mathrm{xy}, \mathrm{q}), \quad \mu_{\mathrm{A}}^{+}\right. \\
& (\mathrm{y}, \mathrm{q})\} \geq \min \left\{\min \left\{\mu_{\mathrm{A}}^{+}(\mathrm{xy}, \mathrm{q}) \mathrm{z}, \quad \quad \mu_{\mathrm{A}}^{+}(\mathrm{z}, \mathrm{q})\right\}, \mu_{\mathrm{A}}^{+}\right. \\
& (\mathrm{y}, \mathrm{q})\} \\
& \geq \min \left\{\operatorname { m i n } \left\{\mu_{\mathrm{A}}^{+}(0, \mathrm{q}), \mu_{\mathrm{A}}^{+}(\mathrm{z}, \mathrm{q}),\right.\right. \\
& \mu_{\mathrm{A}}^{+} \\
& (\mathrm{y}, \mathrm{q})\} \geq \min \left\{\mu_{\mathrm{A}}^{+}(\mathrm{y}, \mathrm{q}), \mu_{\mathrm{A}}^{+}(\mathrm{z}, \mathrm{q})\right\} \\
& \text { (ii) } \mu_{\overline{\mathrm{A}}}(\mathrm{x}, \mathrm{q}) \quad \leq \max \left\{\mu_{\overline{\mathrm{A}}}(\mathrm{xy}, \mathrm{q})\right. \\
& \mu_{\mathrm{A}} \\
& (\mathrm{y}, \mathrm{q})\} \leq \max \left\{\max \left\{\mu_{\overline{\mathrm{A}}}(\mathrm{xy}, \mathrm{q}) \mathrm{z}, \mu_{\overline{\mathrm{A}}}(\mathrm{z}, \mathrm{q})\right\}, \mu_{\overline{\mathrm{A}}}(\mathrm{y}, \mathrm{q})\right\} \\
& \leq \max \left\{\max \left\{\mu_{\overline{\mathrm{A}}}(0, \mathrm{q}), \mu_{\overline{\mathrm{A}}}(\mathrm{z}, \mathrm{q}), \mu_{\overline{\mathrm{A}}}(\mathrm{y}, \mathrm{q})\right\}\right. \\
& \leq \max \left\{\mu_{\mathrm{A}}(\mathrm{y}, \mathrm{q}), \mu_{\mathrm{A}}(\mathrm{z}, \mathrm{q})\right\} .
\end{aligned}
$$

\section{CONCLUSION}

In this present paper, we have presented some properties of interval-valued bipolar fuzzy lattices (IVBQFL) over bipolar Q-fuzzy d-ideals. It is clear that the most of the results can be simply extended to interval-valued bipolar Q-fuzzy lattices / ideals. The obtained results probably can be applied in various fields such as artificial intelligence, signal processing, multi agent-systems, robotics, genetic algorithms, decision making, automata theory and medical diagnosis. It is our hope that this work would serve as a foundation of furthermore study of the theory of soft algebra especially soft ideals. In our opinion the established results can be similarly extended to groups, rings and its generalizations, soft computing and applied in engineering problems.

\section{REFERENCES}

[1] Atanassov, K.T., and Gargov, G. 1989. Interval-valued Intuitionistic fuzzy sets, Fuzzy sets and systems, 31:343349 .

[2] Deschrijver, G. 2007. Arithmetric operators in intervalvalued fuzzy theory, Information sciences, 177:29062924

[3] D. Dubois and H. Prade, Fuzzy sets and systems : Theory and applications, Academic press, 1980.

[4] Gotzalczany, M.B. 1987. A method of inference in approximate reasoning based on interval-valued fuzzy sets, Fuzzy sets and systems, 31:243-251.

[5] Y.B. Jun and S.Z. Song, Subalgebras and closed ideals of $\mathrm{BCH}$-algebras based on bipolar-valued fuzzy sets, Sci. Math. Jpn. (to appear).

[6] K.M. Lee, Bipolar-valued fuzzy sets and their operations, Proc. Int. Conf. On Intelligent Technologies, Bangkok, Thailand (2000), 307-312.

[7] K.M. Lee, Comparison of interval valued fuzzy sets, intuitionistic fuzzy sets, and bipolar valued fuzzy sets, J. Fuzzy Logic intelligent systems 14 (204), no.2, 125-129.

[8] Muhammad Akram., and Wieslaw. A Dudek., Interval Valued intuitionistic fuzzy Lie ideals of Lie algebras, World Applied Sciences Journal, 7(7): 812-819 (2009).

[9] Turksen, I.B. 1986. Interval-valued fuzzy sets based on normal forms, fuzzy sets and systems, 20:191-210.

[10] L.A. Zadeh, Fuzzy sets, Information and control 8 (1965), 338-353.

[11] Zadeh, L.A. 1975. The concept of linguistic variable and its application to approximate reasoning, information science, 8:199-249. 